\title{
Object identification: The mental representation of physical and conceptual attributes
}

\author{
STEPHANIE KELTER, HOLGER GRÖTZBACH, RALF FREIHEIT, \\ BARBARA HÖHLE, SABINE WUTZIG, and EUGEN DIESCH \\ Technische Universität Berlin
}

\begin{abstract}
In two experiments, subjects indicated whether two pictures of familiar objects were equivalent. The picture pairs were identical, showed the same object in various perspectives and states, or showed different objects with varying degrees of conceptual relatedness. In Experiment 1 , the equivalence criterion for judging the picture pairs was varied between subjects (identity of pictures; conceptual equivalence of objects at subordinate, basic, or superordinate level). The reaction times of the four subject groups suggest that a pictorial stimulus is not always mentally represented in the same way, and that the instructions given determine which attributes of the stimulus are represented. In Experiment 2, the visual similarity of the picture pairs was varied. The results indicate, at least with a basic-level equivalence instruction, that not only perceptual, but also non-perceptual, functional attributes are represented-namely, those that, according to Rosch et al. (1976), are common to the members of a superordinate category.
\end{abstract}

Object identification is a quasi-automatic, virtually instantaneous process that is fairly inaccessible to introspection. It is one of the most ubiquitous, if not basic, processes of cognition and a component process of any more complex action. Object identification may not, however, be a unitary process. Presumably, object identification comprises several subprocesses of information transformation, reduction, and elaboration interrelating different mental codes representative of different forms of identification.

In recent years, a number of investigators have made use of picture-matching tasks to study the process of object identification. The experimental paradigm used originated from Posner and Mitchell's (1967) influential study of letter matching. Drawing from this research. Bartram (1976) and Klatzky and Stoy (1974) presented their subjects with same-different picture-picture matching tasks and asked them to judge whether both pictures showed objects from the same (conceptual) category. "Same" responses to pairs of identical pictures and to pictures that were mirror images of each other were faster than "same" responses to pairs of pictures representing the same object from different perspectives. Reaction times (RTs) in the latter condition, in turn, were shorter than RTs in the so-called "name-same" condition, in which the pictures of a pair represented different objects from the same conceptual category

Experiment 2 is part of a larger research project supported by the Deutsche Forschungsgemeinschaft. We wish to thank Iain Boyd Whyte for his invaluable support and assistance with the English text. Requests for reprints should be sent to Stephanie Kclter, Institut für Psychologie, Technische Universität Berlin, Dovestrasse 1-5, 1000 Berlin (West) 10, West Germany.

-Steven W. Keele served as Action Editor for this manuscript. (e.g., two different bridges). Results were interpreted as reflecting different levels of processing at which a match of the two stimuli is obtained. Bartram $(1974,1976)$ suggested that there are at least three levels of coding: (1) a perspective-bound "picture code," which is a more or less veridical representation of the pictorial stimulus, (2) a more abstract "object code," which is specific to the particular object presented, but is independent of a particular perspective, and (3) a "semantic code," which represents the depicted object as a member of a conceptual category.

Bartram $(1974,1976)$ conceded that this hypothetical sequence of three coding levels may be a considerable oversimplification. It is indeed implausible that there should be no more than one level of conceptual representation. Identification implies categorization and categorization develops into fully fledged taxonomies. A chair may be just a chair in one situation, a kitchen chair in a second, and a piece of furniture in still another. Now, are the different conceptual levels assigned to different stages in the identification process? And if so, what is the order of stages?

Rosch and her collaborators (e.g., Mervis \& Rosch, 1981; Rosch, 1975; Rosch, Mervis, Gray, Johnson, \& Boyes-Braem, 1976) have shown in numerous experiments that there is a distinct level of abstraction in natural taxonomies-the "basic" level-at which the categories are at their most informative and most differentiated from each other. Their studies suggest that objects are categorized at this basic level by default. According to Rosch (1978), the basic-level superiority is already given in the process of object identification: "objects may be first seen or recognized as members of their basic category, and ... only with the aid of addi- 
tional processing can they be identified as members of their superordinate or subordinate category" (pp.34-35). Similar proposals have been put forward by Fraisse and his colleagues (Fraisse, Lanati, Régnier, \& Wahl, 1965; Segui \& Fraisse, 1968), Hoffmann (1980), and Seymour (1973). Until now, however, the empirical evidence for this claim was derived from naming tasks and pictureword matching tasks only. No clear conclusions about object identification can be drawn, however, from experiments such as these. One reason for this is the correlation that exists between the various word characteristics (e.g., word length, word frequency) and the subordinate, basic, and superordinate levels. These verbal factors might account for the basic-level superiority attained in those studies (see Murphy \& Smith, 1982, for a discussion of this problem). Second, various studies have indicated that the semantic system (the representation of the meaning of words) and the conceptual system (the representation of nonverbal meaningful stimuli) are by no means identical (Durso \& Johnson, 1979; Guenther \& Klatzky, 1977; Guenther, Klatzky, \& Putnam, 1980; Warren \& Morton, 1982). It is unclear, therefore, whether word-picture matching tasks or naming tasks actually reflect the process of object identification or merely the "translation" between semantic and conceptual representations.

In the present study, the "basic-level-first" hypothesis of object identification was tested by means of an entirely nonverbal task, namely, the picture-picture matching task of Bartram (1976) and Klatzky and Stoy (1974). Items representing conceptual equivalence were differentiated according to the various levels of the conceptual hierarchy. Accordingly, six item types of picture pairs were used. In addition to the usual "same picture" (PIC) and "same object from different perspectives" (OBJ), four other types were shown: SUB-two different objects from the same subordinate category (e.g., two different kitchen chairs); BASIC-two objects from different subordinate categories but the same basic category (e.g., an office chair and a kitchen chair); SUPER-two objects from different basic categories but the same superordinate category (e.g., a bed and a kitchen chair); DIFF-two objects from different superordinate categories (e.g., a cigarette and a kitchen chair).

The equivalence criterion to be employed by the subjects during the test was also varied in a way similar to the way in which the item types ware varied. One subject group had to indicate equivalence on subordinate category level, another group equivalence on basic category level, and a third group equivalence on superordinate category level. A fourth group, also included, was instructed merely to indicate whether or not the pictures of a pair were (physically) identical. From this group we hoped to obtain some information about the extent to which pictures are encoded conceptually even when the task requires no conceptual processing. There has been some evidence for "shallow" nonconceptual pro- cessing of visual stimuli under such instructions (Rosch, 1975, Experiment 4).

Assuming that the RTs reflect the encoding stages in such matching tasks and following the "basic-levelfirst" hypothesis, we would generally expect comparatively short RTs in all those cases in which an identification at the basic level would be sufficient to solve the task. Longer RTs would be expected when the subject had to determine the categorical membership at the subordinate or superordinate level before a decision about the equivalence of the pictures could be reached. More specifically, one would expect that:

1. Subjects instructed to establish equivalence on the basic level would judge the SUB and BASIC items equally quickly as "equivalent," and likewise the SUPER and DIFF items equally quickly as "nonequivalent," because in each case, the decision could be based solely on the identification of the objects at the basic level.

2. With a superordinate-level instruction, one would expect the same short RTs with SUB and BASIC items, but longer RTs with SUPER items, because an additional step after the basic-level identification would be needed here before the equivalence of the objects could be established.

3. With a subordinate-level instruction, delayed responses would be expected with the SUB and BASIC items, because in these cases the subjects would have to reevaluate the stimuli, which would have been initially recognized as basic-same, in order to establish whether they were equivalent at the subordinate level. That is to say: First, the increase in "same" RT from the "neutral" PIC and OBJ conditions to the special SUB condition should be particularly large in this group when compared with those in the other groups, and second, the RTs of negative responses to the BASIC items should be larger than those to the SUPER or DIFF items, for which the mismatch at the first coding stage would stimulate the negative response.

It should be emphasized that all these predictions are valid only when one makes the usual assumption that the variation of the RT in matching tasks like these reflects the different levels of processing at which a match of the two stimuli is obtained. As we shall see, this assumption is not entirely unproblematical.

\section{EXPERIMENT 1}

\section{Method}

Subjects. Forty-ight male and female students at the Technische Universität Berlin served as volunteer subjects. They were assigned randomly to one of four groups of 12 subjects each.

Materials. Stimuli were $36 \times 24 \mathrm{~mm}$ color slides of familiar objects. The objects were photographed against a beige background, and both large and small objects were approximately the same size on the slides. Each item was made up of two pictures, a prime picture and a test picture. Two item pools were prepared. Items from the first pool were used in the experimental trials; items from the second pool were used in warmingup and filler trials. For the pool of experimental items, there 
were 18 test pictures, each showing an object from a different superordinate category. For each test picture, 6 alternative prime pictures were prepared, defining six different item types: (1) a prime picture identical to the test picture (PIC), (2) a prime picture showing the same object as the test picture, but from a different perspective $(\mathrm{OBJ}),(3)$ a prime picture showing a different object from the same subordinate category (SUB), (4) a prime picture showing a different object from the same basic category, but in a different subordinate category (BASIC). (5) a prime picture showing a different object in the same superordinate category, but from a different basic category (SUPER), and (6) a prime picture showing an object from a different superordinate category (DIFF).

The levels of equivalence in the item types PIC and OBJ were drawn from Bartram (1976). The levels of equivalence in the item types SUB, BASIC, SUPER, and DIFF were defined in accordance with the system of classification proposed by Rosch et al. (1976). From the 18 test pictures and their respective sextuplets of prime pictures, altogether 108 experimental item: were constructed.

The item pool for warming-up and filler trials comprised 70 items. Twenty-one items represented the relation of type PIC, 10 of type OBJ, 5 of type SUB, 5 of type BASIC, 8 of type SUPER, and 21 of type DIFF. The objects used in these items were drawn from superordinate categories different from those used in the experimental items. The filler items were used to balance the proportion of positive and negative trials within each subject (see below).

Finally, an additional five item sextuplets (i.e., 30 items) were prepared for use in the instruction phase of the experiment. Again, the categories of these items differed from those used in the other item pools.

Apparatus. Stimulus slides were projected onto a screen approximately $230 \mathrm{~cm}$ in front of the subject. The projected picture measured about $72 \times 48 \mathrm{~cm}$. Two Kodak Carousel 2020 projectors were used, one for the prime and one for the test pictures. Both projectors were fitted with a Compur shutter, which was controlled by a Hewlett-Packard computer. RTs were measured by the computer's internal timer, which was started simultaneously with the onset of the test picture and was stopped by the subject's pressing a response key. The "same" response key was assigned to the subject's right hand and the "different" response key to the subject's left hand.

Design and Procedure. Four groups of 12 subjects each were run. One group was instructed to give a "same" response if the prime and the test picture in the trial were identical and a "different" response if they were not ("picture-level instruction group"). A second group was instructed to indicate whether the object(s) shown in the two stimuli came from the same subordinate category ("subordinate-level instruction group") The third group was instructed to indicate whether they came from the same basic category ("basic-level instruction group") and the fourth group was instructed to indicate whether they came from the same superordinate category ("superordinatelevel instruction group"). All subjects were presented with al the six item types described above (PIC, OBJ, SUB, BASIC, SUPER, and DIFF). This resulted in a $4 \times 6$ factorial design with instruction group as the between-subjects factor and item type as the within-subjects factor.

Each subject received 48 items. The first 18 items of the experiment served as warming-up trials. In the remaining 30 trials, 18 experimental and 12 filler trials were presented in a mixed random order, which was different for each subject. The test pictures of the 18 experimental items were the same for al subjects. From the experimental item pool, prime pictures of the 18 test pictures were selected for each subject in such a way that 3 items represented item type PIC, another 3 item type OBJ, and so on. Within each instruction group, two subjects received a given test picture with the associated PIC-prime preceding it, another two subjects received the same test picture with the associated OBJ-prime preceding it, etc. Thus, within and across the instruction groups, all subjects were given the same test pictures, and all prime pictures occurred with the same frequency. Filler and warming-up items were selected such that the proportion of positive and negative trials was balanced for each subject. For instance, the subjects of the basic-level instruction group, for whom 12 experimental trials were positive and 6 negative, received 3 positive filler trials (1 OBJ-filler, 1 SUBfiller, and 1 BASIC-tïller), and 9 negative filler trials ( 5 SUPERfiller and 4 DIFF-filler).

The procedurc for each trial was as follows: The prime picture was projected for $500 \mathrm{msec}$. After an interstimulus interval of $500 \mathrm{msec}$, the test picture was presented for $1,000 \mathrm{msec}$. The subjects were to press one of the two keys immediately to indicate whether or not the stimuli were equivalent. An intertrial interval of $5,000 \mathrm{msec}$ followed before a new trial was started automatically

The subjects were tested individually in a dimly illuminated room. They were told that they would be presented with pairs of pictures, and were instructed to press the "same" or "different" response key as fast as possible, depending on whether the pictures were equivalent or not. The subjects were familiarized with the respective criterion of equivalence by means of a series of 18 items drawn from three item sextuplets. To ensure that the subjects comprehended the task, 12 practice trials drawn from another two sextuplets were given.

\section{Results}

Of the total of 864 responses to the experimental items, 57 were errors $(6.6 \%)$, of which 24 were false positives and 33 were false negatives. Error rates were generally positively correlated with the mean RT over the variables analyzed. This contraindicates a speedaccuracy tradeoff. Errors were discarded from the results. The mean RT of correct responses in every condition was determined for each subject. These scores were then submitted to a repeated measures ANOVA separately for each instruction group and for positive and negative trials. For a posteriori comparisons, NewmanKeuls tests were used (Winer, 1970).

Figures 1 to 4 show the mean RTs for the positive and negative responses for the four instruction groups, respectively. For the picture-level instruction group, mean RT of "same" responses (item type PIC) was 497 msec. Mean RTs of "different" responses ranged from 611 to $492 \mathrm{msec}$ for the remaining five item types. The difference within them was significant $[\mathrm{F}(4,44)=4.28, \mathrm{MSe}=6,236, \mathrm{p}<.01]$. Pairwise comparisons showed that the RTs under the BASIC, SUPER, and DIFF conditions were significantly shorter than the RTs under the OBJ condition $(\mathrm{p}<.05)$. All other differences did not reach significance. For the subordinate-level instruction group, the effect of the item type proved to be significant, both for the "same" responses $[F(2,22)=21.73, \mathrm{MSe}=37,700, \mathrm{p}<.01]$ and for the "different" responses $[\mathrm{F}(2,22)=8.87, \mathrm{MSe}=$ $40,149, \mathrm{p}<.01]$. "Same" responses under the PIC and the OBJ conditions were faster than under the SUB condition $(p<.05)$. "Different" responses under the BASIC condition were slower than under the SUPER and the DIFF conditions $(p<.05$ ). For the basic-level instruction group, the difference between the four posi- 


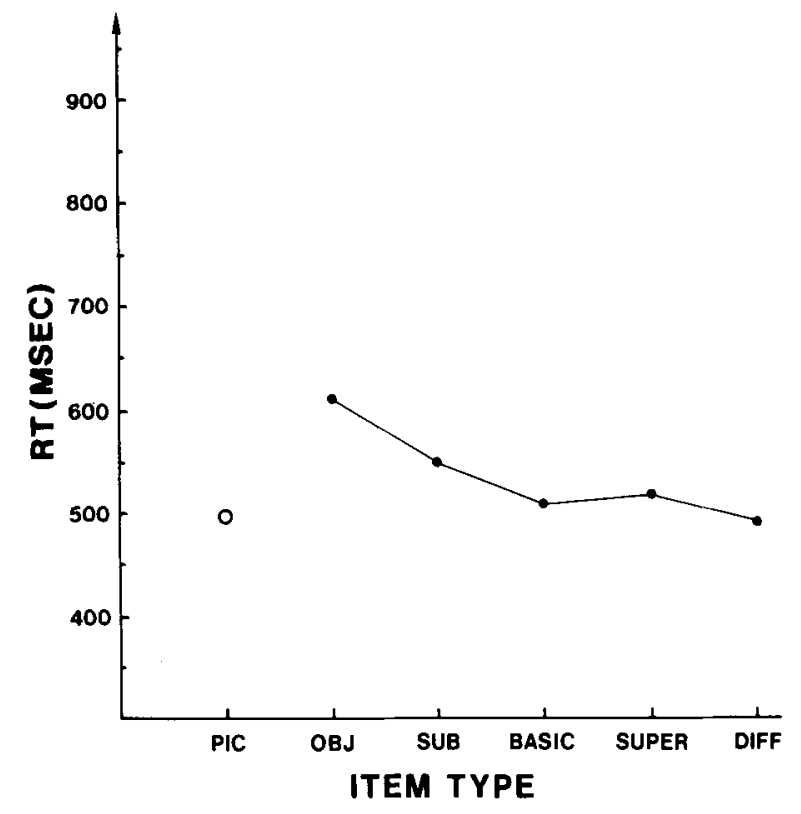

Figure 1. Picture-level instruction group: Mean RT (in milliseconds) as a function of item type for "same" responses (unfilled circles) and "different" responses (filled circles).

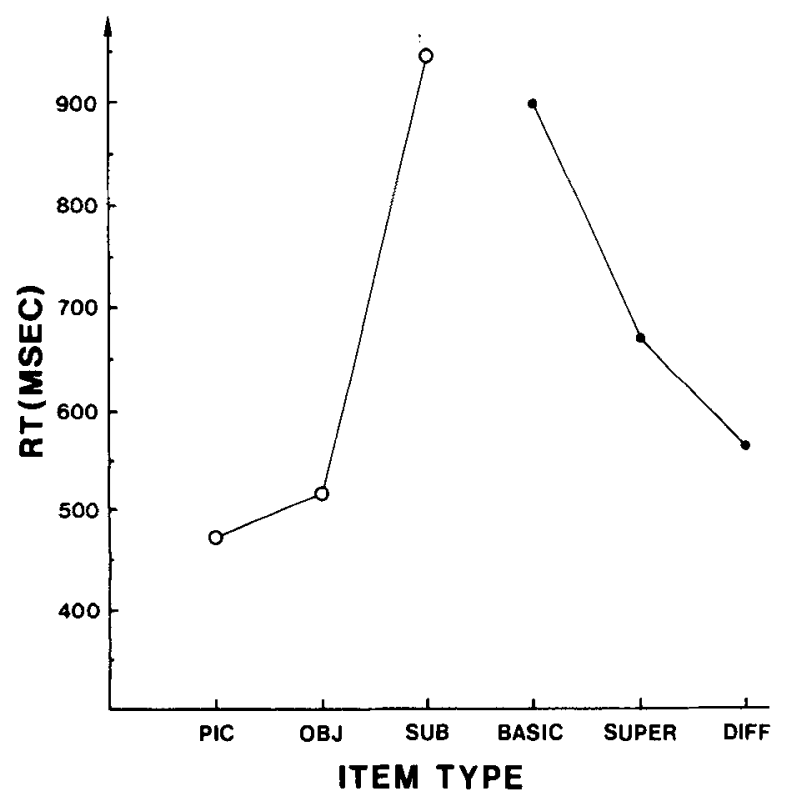

Figure 2. Subordinate-level instruction group: Mean RT (in milliseconds) as a function of item type for "same" responses (unfilled circles) and "different" responses (filled circles).

tive conditions (PIC, OBJ, SUB, and BASIC) was significant $[F(3,33)=10.14, \mathrm{MSe}=19,965, \mathrm{p}<.01]$. A posteriori tests revealed that the RTs under the SUB condition were significantly longer than the RTs under the PIC condition, and that the RTs under the BASIC condition were longer than the RTs under the PIC and the OBJ conditions. Furthermore, the difference of main interest between BASIC RTs and SUB RTs was

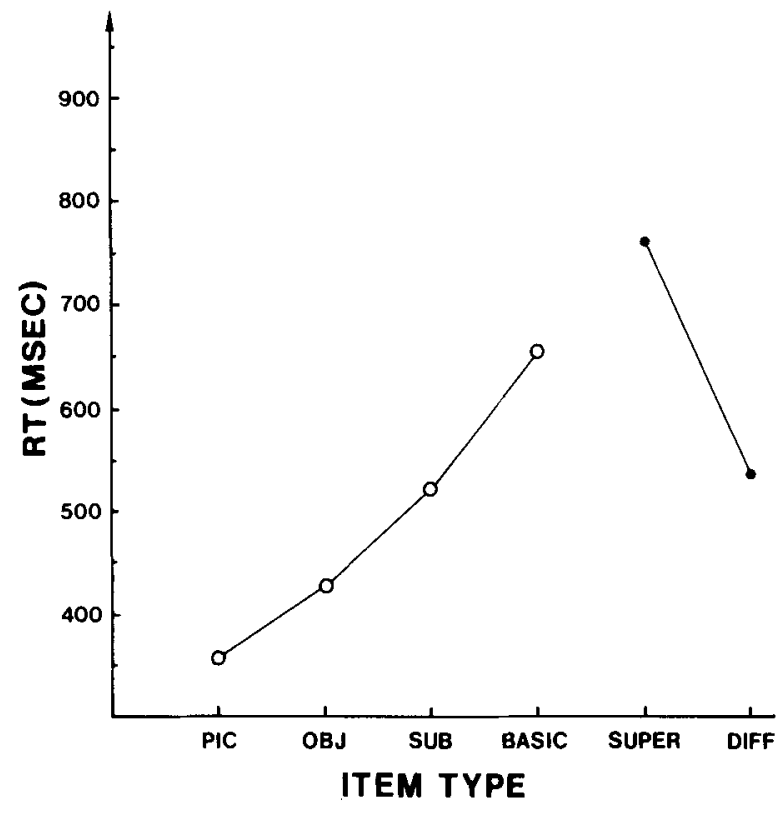

Figure 3. Basic-level instruction group: Mean RT (in milliseconds) as a function of item type for "same" responses (unfilled circles) and "different" responses (filled circles).

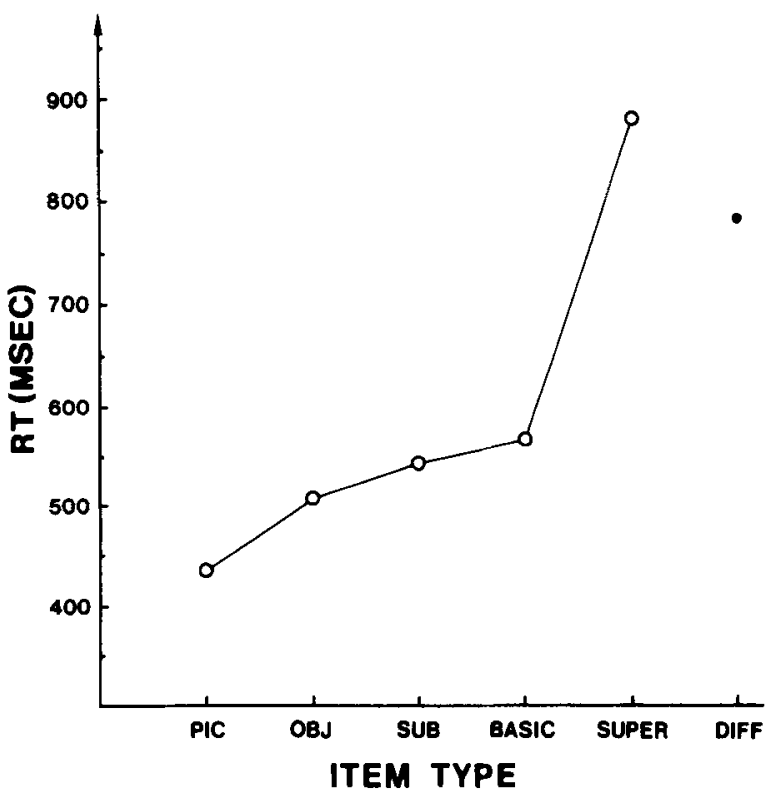

Figure 4. Superordinate-level instruction group: Mean RT (in milliseconds) as a function of item type for "same" responses (unfilled circles) and "different" responses (filled circles).

also significant $(\mathrm{p}<.05)$. "Different" RTs proved significantly longer under the SUPER condition than under the DIFF condition $[\mathrm{F}(1,11)=5.31, \mathrm{MSe}=56,048$, $\mathrm{p}<.05]$. For the superordinate-level instruction group, too, the difference between the positive conditions (PIC, OBJ, SUB, BASIC, and SUPER) was significant $[F(4,44)=7.90$, MSe $=44,801, p<.01]$. SUPER RTs were significantly longer than the RTs under the PIC, 
OBJ, SUB, and BASIC conditions $(p<.05)$; the latter mentioned conditions did not differ significantly from each other in pairwise comparisons. Mean RT of "different" responses under the DIFF condition was $788 \mathrm{msec}$.

To test the prediction, as described in Point 3 of the introduction, the significance of the interaction between the factors "instruction group" and "item type" was tested by means of a two-way ANOVA. To avoid mixing RTs from positive and negative trials, only the data from the subordinate-, basic-, and superordinate-level instruction groups was used. The item types PIC, OBJ, and SUB represented the three levels of the second factor. The analysis yielded a significant main effect of both factors $[F(2,33)=3.81, \mathrm{MSe}=109,356, p<.05$ and $\mathrm{F}(2,66)=34.32, \mathrm{MSe}=17,704, \mathrm{p}<.01$, for "in struction group" and "item type," respectively]. The interaction also proved significant $[\mathrm{F}(4,66)=9.39$. MSe $=17,704, p<.01]$. Post hoc tests showed that the effect of the instructions was significant only under the SUB condition $[\mathrm{F}(2,33)=14.02, \mathrm{MSe}=48,254, \mathrm{p}<$ .01], but not under the PIC or the OBJ condition $[F(2,33)=0.90$, and $F(2,33)=0.59$, respectively $]$. According to the Newman-Keuls test, the RTs under the SUB condition were significantly longer with a subordinate-level instruction than with a basic-level or superordinate-level instruction $(\mathrm{p}<.01)$. The difference between the latter mentioned instructions was not significant.

\section{Discussion}

In the instances in which our experiment corresponds in its conditions to the experiments of Bartram (1976) and Klatzky and Stoy (1974), the earlier results were basically confirmed: In the subject groups with a "conceptual" equivalence criterion, RTs were at their shortest when the two pictures were identical. RTs were longer when the same object was shown in different perspectives, and they were at their longest when two different objects from the same conceptual category were shown.

With regard to our main objective, which was to establish how objects are conceptually identified, a less clear picture emerged. It is true that the results for the subordinate- and superordinate-level instruction groups agree well with the "basic-level-first" hypothesis. The subordinate-level instruction group showed, on the one hand, a disproportionately large increase in RT from the "neutral" PIC and OBJ conditions to the SUB condition, and, on the other hand, a significant delay in the negative responses under the BASIC condition when compared with the SUPER and DIFF conditions. Similarly, with the superordinate-level instruction group, the RTs under the SUPER condition were, as expected, longer than under the BASIC or SUB condition. But the results for the basic-level instruction group are less consistent with the "basic-level-first" hypothesis. The fact that the RTs under the SUB condition were signifi- cantly shorter here than under the BASIC condition suggests that, against our expectations, the SUB-same items might have been recognized as equivalent at an earlier stage in the identification process than the BASIC-same items. It is conceivable that the visual similarity of the SUB items was so great that, in some cases, the subjects judged the objects to be equivalent at Bartram's second coding level, namely, the objectcode level. In this case, however, one would also have expected shortened RTs from the superordinate-level instruction group with the SUB items. In fact, the difference here between the SUB and BASIC conditions was only slight and statistically insignificant. Also incompatible with the "basic-level-first" hypothesis is the fact that, with the basic-level instruction group, the RTs under the SUPER condition were significantly longer than under the DIFF condition. This negative-relatedness effect must raise doubts about the presuppositions behind our experiment. These doubts are reinforced if one considers the RT profiles as a whole because there is a more or less strongly marked negative-relatedness effect with all groups. One should remember that the RTs in matching tasks like ours were generally considered to be indications of the number of stages or "depth" of processing: If RT is shorter under one condition than under another, then it is concluded that the match is attained at an earlier stage under the one condition than under the other. It is assumed that the subject proceeds through a succession of encoding stages. At each stage, the subject checks whether or not the mental representations of the two stimuli are equivalent. If they are equivalent, the subject gives a positive response; if they are not equivalent, the subject proceeds to the next stage, until that coding level that corresponds to the equivalence criterion prescribed by the instruction is eventually reached. If the mental representations still do not match at the level of equivalence criterion, the subject gives a negative response. In the past, research has focused on the RTs of "same" responses. But for the validity of the model, the RTs in negative trials are equally important. By proceeding from a self-terminating process (assuming that the encoding process stops when the level of the equivalence criterion is reached), the stage model implies that the $\mathrm{RT}$ of negative responses cannot be affected by stimulus equivalence "beyond" the equivalence criterion. Thus, the negative relatedness effect observed in our data does not specifically contradict the "basic-level-first" hypothesis, but more generally indicates that all stage models of the type outlined above are inadequate for our dataand perhaps not only for our data: Murphy and Smith (1982) also obtained a negative-relatedness effect with their word-picture matching task. As these authors correctly pointed out, it follows that the stage model must be wrong and that the RT variations across positive conditions obtained in the earlier studies with wordpicture matching tasks cannot be accepted as proof that 
more encoding stages must be gone through in subordinate or superordinate categorization than in basic categorization.

Proctor (1981) recently suggested a model for matching tasks in which the RTs are not taken to reflect the number of encoding stages, but the speed of the encoding processes. In Proctor's view, RT differences in sequential matching tasks reflect the extent to which the first stimulus produces "activation that results in a more rapid encoding of the second stimulus when it is the same as the first" (p. 322). Proctor's argument is convincing for the data that he presents. He does not, however, consider RTs from negative trials. Indeed, his proposal cannot explain a negative-relatedness effect, unless one assumes that the encoding of the second stimulus is accelerated more by a different prime (as in our DIFF condition) than by a semantically or conceptually related prime (as in our SUPER condition).

\section{The "Distance-From-the-Cutoff-Point" Model}

A considerable amount of research has been done in various areas of experimental psychology on the negative-relatedness effect, for example, in relation to verifying category membership (e.g., McCloskey \& Glucksberg, 1979; Murphy \& Smith, 1982; Schaeffer \& Wallace, 1970; Smith, Shoben, \& Rips, 1974), comprehension of antonymy (Herrmann, Chaffin, Conti, Peters, \& Robbins, 1979) or matching letter strings (e.g., Krueger, 1978; Ratcliff, 1981). Irrespective of the considerable differences that exist between the various models suggested by the authors listed above, in all of them the variation of the RT dependent on relatedness is attributed to the comparison and/or decision processes, not to the encoding processes. Applying the basic prin. ciples of the most refined models of McCloskey and Glucksberg (1979), Krueger (1978), and Ratcliff (1981) to our matching task, one may suggest that the duration of the comparison/decision processes, and therefore of the RT, depends on at least the following two parameters: (1) the global similarity between the two mental representations of the two stimuli to be compared, whereby the "global similarity" may be conceived of as the amount of feature overlap or as some inverse monotonic function of the mental distance of the stimuli (cf. Hutchinson \& Lockhead, 1977), and (2) the position of the critical cutoff point(s) on an internal scale of similarity. The position of the critical cutoff point is determined by, among other factors (e.g., response strategies), the instructions, which specify when the responses "same" and "different" are to be given. If the actual similarity shared by the mental representations of a given pair of stimuli is below the critical cutoff point, the subject gives a negative response; if the similarity is above the critical cutoff point, the subject gives a positive response. The RT is assumed to be some inverse monotonic function of the distance between the actual similarity of the mental representations and the cutoff point; that is, the greater the distance on the scale of similarity, the shorter the RT, and vice versa. Applying this simple principle, it is easy to explain the negative-relatedness effect: Two very different stimuli have a similarity value that is much further from the cutoff point than two different, yet relatively similar, stimuli.

Our data, both from the negative and positive trials, conform quite closely to this "distance-from-the-cutoffpoint" model. The only assumption that one must make is that, in our Experiment 1, the similarity of the mental representations of the two stimuli making up a pair decreases from the PIC condition via conditions OBJ, SUB, BASIC, and SUPER to condition DIFF. In all our subject groups, the RT increased initially until the cutoff point defined by the respective instruction was reached and then decreased through the negative conditions. This is exactly what the "distance-from-the-cutoff-point" model implies.

According to this interpretation, the RT in matching tasks like ours does not provide information about the procedural aspects of object identification (i.e., about the number or duration of the encoding stages), but does provide information about the subjective similarity between the mental representations to be compared. But one should bear in mind that this interpretation might also be based on an oversimplification. Schvaneveldt, Durso, and Mukherji (1982) produced evidence that suggests that, at least with verbal categorization tasks, the semantic relatedness of the stimuli affects both the encoding processes (lexical access) and also the comparison/decision processes, so that the RTs in tasks like these reflect, in each case, a mixture of these two effects.

What conclusions can now be drawn if our data are interpreted in terms of the "distance-from-the-cutoffpoint" model? In order to answer this question, we should recapitulate how the six picture conditions that we used differed from each other. Under the DIFF condition, pictures with a minimum of similarity (both physical and conceptual) were paired together. The pictures that were to be compared under the SUPER condition had a higher degree of similarity, if only in particular respects. According to Rosch et al. (1976), the members of a superordinate category have only their functions in common; the correlation of the other attributes of the category members, for example, the visual attributes, is still insignificant. However, at the basic level, the members of a given category have in common the same general shape (Rosch et al., 1976) and a substantial number of parts (Hemenway, 1981, as cited in Murphy \& Smith, 1982, p. 19). Thus, one may assume that the pictures paired together in the BASIC condition shared not only functional attributes, but also many visual attributes. Such correspondences presumably appeared even more frequently with the picture pairs of the SUB condition. It should be mentioned that these correspondences concern the conceptually relevant 
aspects of the objects (e.g., the handle of a frying pan). There may also be occasional correspondences between conceptually irrelevant aspects of the objects. In our material, these first appear in significant numbers in the OBJ and PIC conditions. Under these two conditions, the pictures making up a pair match, by definition, not only in their conceptually relevant aspects, but also in many, somtimes in all, of their idiosyncratic aspectsthat is, aspects that are specific to the particular object.

With the help of the new interpretative framework, one can now approach the question of whether the subjects in our tasks do or do not mentally represent all the attributes of the objects (i.e., the functional, visualconceptual, and idiosyncratic attributes). First, if the subject has represented the functional attributes, then the RT under the conditions SUPER and DIFF should be different. If, however, the functional attributes have not been mentally represented, then no difference between these two conditions should be expected, because the subjective similarity between pairs of pictures under the SUPER condition is then no greater than the similarity of the picture pairs of the DIFF type. Second, it is possible, by comparing the RTs under the BASIC and SUPER conditions, to ascertain whether or not the subjects code the visual-conceptual attributes of an object. Third, a comparison of the RTs under conditions OBJ and SUB tells us whether or not the idiosyncratic, conceptually nonrelevant aspects are also mentally represented. This is so because conditions SUB and OBJ (or PIC) differ from each other only in as far as the two pictures of an item concur or differ in their idiosyncratic, conceptually irrelevant aspects.

Let us no look at the results obtained from each particular group of subjects. It would seem that virtually no conceptual coding of the stimuli tonk place in the picture-level instruction group: There is no evidence of coding the visual-conceptual attributes (BASIC vs. SUPER, n.s.) or the functional attributes (SUPER vs. DIFF, n.s.), although caution must be exercised when interpreting a null effect. The subordinate-level instruction group also represented the idiosyncratic attributes of the objects (OBJ vs. SUB, significant). Their mental representations did, however, already include the visual conceptual attributes (BASIC vs. SUPER, significant). No clear conclusions can be drawn from our data with regard to the functional attributes-the difference between SUPER and DIFF just missed the 5\% level of significance. In contrast, in the basic-level instruction group, the difference between the SUPER and the DIFF conditions proved significant, indicating that these subjects did mentally represent the functional attributes of the objects. It seems, however, that even this subject group failed to fully abstract from the idiosyncratic aspects of the stimuli (OBJ vs. SUB, n.s., but PIC vs. SUB, significant). Finally, in the superordinate-level instruction group, the only significant difference was obtained between the BASIC and SUPER condition. This suggests that only the conceptually relevant characteristics of the pictures were represented mentally.
Thus, we are able to establish that the subjects used different mental representations according to the instruction given. It follows that it is pointless to speak of the mental representation of pictures as a constant and uniform code. One may argue that the varying results for the four instruction groups do not reflect real differences in coding strategies, but merely show that each group initiated the processes of comparison and decision at a different point in time. For example, the picture-level group might have begun with the comparison and decision processes before they had represented the conceptual aspects, whereas the superordinate-level group might have begun after they had separated the conceptually relevant from the conceptually irrelevant aspects. However, the similar overall levels of the RT profiles of the various instruction groups do not support this interpretation.

Our results also show that the mental representations of the stimuli were by no means "tailor-made" for the various tasks. Generally, the subjects included more aspects in their representations than were necessary to solve the task. The superordinate-level instruction group did not abstract from the visual-conceptual attributes, and the subjects in the basic-level and superordinatelevel instruction groups did not abstract from the idiosyncratic elements. Only when the task demanded no conceptual coding whatsoever, as with the picturelevel instruction group, did the subjects represent only those attributes demanded by the task.

To our knowledge, the task specificity of the mental coding of pictorial stimuli has not, with the exception of learning tasks (e.g., Babbitt, 1982; Frost, 1972), been investigated systematically (for verbal stimuli see, e.g., Barsalou, 1982, and Kroll, Kellicut, Berrian, \& Kreisler, 1974). Nevertheless, there are some findings of other authors with which certain aspects of our results might be compared. For example, Rosch (1975) found that semantic priming facilitated picture matching only when the match criterion was membership of a conceptual category, but not when it was the "physical" identity of the pictures. This result corresponds very well with the total lack of conceptual coding in our picture-level group, in contrast with the three "conceptual-equivalence" instruction groups. The findings of Klatzky and Stoy (1978, Experiment 3) suggest that when a superordinate category term is given as a match criterion, the subjects mentally represent more attributes than are necessary to solve the task. More precisely, they code those "semantic" attributes that characterize the respective basic category, although not the specific details of the respective subordinate category. Again, these results correspond closely to our findings. There are discrepancies, however, between our results and those of Murphy and Smith (1982), who examined the extent to which the functional, nonperceptual attributes of objects are mentally represented. In their study, they employed word-picture matching tasks with artificial subordinate, basic, and superordinate categories and found that the RTs in negative trials remained the same, 
irrespective of any functional similarity between the target word and the picture. It would seem, therefore, that their subjects did not mentally represent the functional attributes. This accords with our own results, as far as the subordinate-level instruction group is concerned. However, Murphy and Smith obtained these negative findings not only for categorization that must be performed at the subordinate level, but also for that which must be performed at the basic level. In our basic-level group, in contrast, we found that the RTs in the SUPER condition were significantly longer than the RTs in the DIFF condition, which indicates that the subjects were mentally representing the functional attributes of the objects depicted. The discrepancy between these two results may derive from the methodological differences between our study and that of Murphy and Smith. It might also be possible, however, that our positive result is nothing but an artifact. Since our study was initially conceived with another purpose in mind, we did not pay particular attention to the visual similarity of the pictures in each pair. Thus, it may be that the pictures paired under the SUPER condition were, by chance, visually more similar than those paired under the DIFF condition, and that the negative-relatedness effect resulted from the differing degrees of visual similarity between the two conditions.

To test this, we conducted a second experiment, in which the visual similarity and the functional correspondence of the picture pairs in the negative trials were varied systematically. Instead of the two item types SUPER and DIFF, we had four: (1) pairs of visually similar pictures showing objects from related conceptual categories, (2) pairs of visually dissimilar pictures showing objects from related conceptual categories, (3) pairs of visually similar pictures showing objects from different superordinate categories, and (4) pairs of visually dissimilar pictures showing objects from different superordinate categories. All subjects were given a basic-level instruction, since in Experiment 1 the only significant difference between SUPER and DIFF occurred with this instruction. In addition, Experiment 2 differed from Experiment 1 in the following respects. First, instead of photographs, drawings were used, because visual similarities were easier to manipulate with drawings. Second, the pictures were not shown one after the other, but simultaneously. In the context of investigating the processing of functional attributes, this seemed to us to be a conservative procedure, in that a simultaneous presentation seemed more conducive to the comparison of the perceptual elements rather than the functional attributes. The third difference between the two experiments concerned the positive conditions: The item type SUB was replaced by an item type STATES (see below). This change had nothing to do with the aim of Experiment 2, for which the positive conditions were of no importance, but stemmed from the intention to reuse the same material in a different study (Kelter, 1982).

\section{EXPERIMENT 2}

\section{Method}

Subjects. Twenty-six subjects took part in the experiment. The subjects had answered advertisements and were paid for their participation. The mean age was 45.6 years $(S D=10.2$; range $=28-60$ ).

Materials. The material comprised 138 slides, each showing two black-and-white drawings of everyday objects, set beside each other. Eighteen of these picture pairs were used for the instruction and warming-up trials. The remaining 120 picture pairs, making up the actual stimulus material, were divided into eight sets, each comprising 15 picture pairs. Four sets comprised pairs of conceptually equivalent pictures; these were (1) pairs of identical pictures (PIC), (2) pairs of pictures showing the same object from different perspectives (OBJ-PERS), (3) pairs of pictures showing the same object in different states (OBJ-STATES), for example, two pictures showing the same umbrella, once opened and once closed, and (4) pairs of pictures showing different objects from the same basic category (BASIC). The four other sets were made up of picture pairs showing conceptually nonequivalent objects. These four sets, which defined the four negative conditions, were (1) pairs of visually similar pictures showing two conceptually related objects $(\mathrm{V}+\mathrm{C}+)$, (2) pairs of visually dissimilar pictures showing conceptually related objects $(\mathrm{V}-\mathrm{C}+),(3)$ pairs of visually similar pictures showing conceptually unrelated objects $(\mathrm{V}+\mathrm{C}-$ ), and (4) pairs of visually dissimilar pictures showing conceptually unrelated objects (V-C--). Examples of the item types for the negative trials are given in Figure 5.

The pictures were selected from a large pool of single drawings. The initial selection process was made by showing the single pictures to 15 students. Of the whole pool, only those pictures that were described by $80 \%$ of the students with the same word were retained for the subsequent stages of selection. The word that emerged at this stage was adopted as the "basiclevel term" for the given drawing. Pairs of different objects with the same basic-level term were then established for the "same". condition BASIC. For the negative conditions $\mathrm{V}+\mathrm{C}+$ and $\mathrm{V}-\mathrm{C}+$, pictures were paired together that did not have the same basiclevel term, but that nevertheless belonged to the same superordinate category or that had significant functional, nonperceptible attributes in common, without sharing the same superordinate term (e.g., backpack and hiking boots). For the negative conditions $\mathrm{V}+\mathrm{C}-$ and $\mathrm{V}-\mathrm{C}-$, pairs were made up of objects that neither came under the same superordinate category nor had any particular situational or functional relationship to each other (e.g., no pairing of an apple and a knife). The differentia-
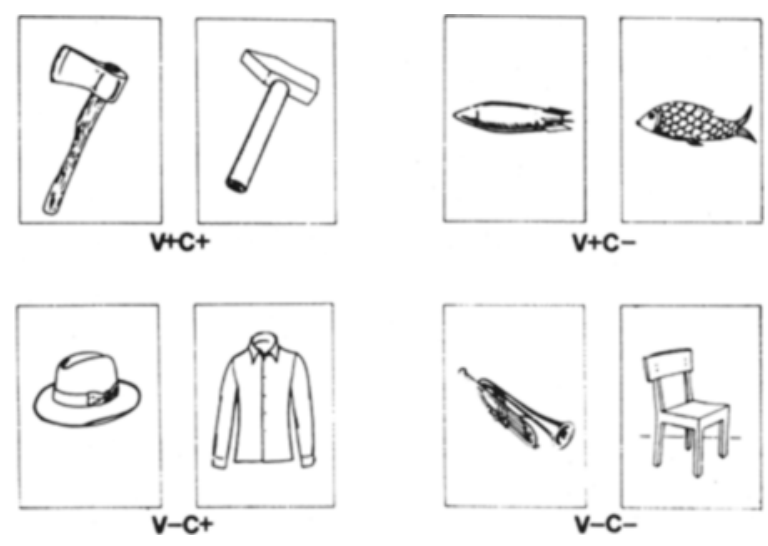

Figure 5. Examples of stimulus pairs used in the negative conditions of Experiment 2. 
tion between visually similar and visually dissimilar pairs was based on the judgment of a further four students, who were asked to choose from the possible pairings described above, those pairs that in their overall shape and shading were most and least similar. In doing this, the raters were instructed to concentrate on the visual appearance rather than on the meaning of the pictures. In this way, sets comprising 17 or 18 picture pairs were assembled for each of the picture conditions. The items required for the instruction and warming-up trials were then selected, so that the remaining 15 picture pairs intended for each condition were comparably familiar. To assess the familiarity of the objects, we used the results of two groups, each of $20 \mathrm{sub}$ jects, who were asked to estimate the word frequency of the basic-level terms of the objects on a 7-point scale.

Apparatus. The stimuli were projected onto a small $(21 \mathrm{x}$ $21 \mathrm{~cm}$ ) screen using a Kodak Carousel A-AV 2030 projector with a built-in shutter. The distance between the subject and the screen was approximately $50 \mathrm{~cm}$. The display of the slides was controlled by a SC/MP microprocessor, which also measured ine RT, that is, the time from the opening of the shutter to the reaction of the subject. The subject responded by pressing one of two keys with the index finger of his or her preferred hand (in all cases, the right hand). The keys were set $4.5 \mathrm{~cm}$ apart on a small box that lay on the table in front of the subject's hand. Fifty percent of the subjects used the right key for a positive response and the left key for a negative response, and the other subjects used the reverse arrangement.

Procedure. The subjects were tested individually. It was explained to them that two pictures would be shown in each trial, and that for each pair they should indicate, by pressing one of the two keys, whether or not the two pictures were conceptually equivalent. The response criterion was indicated by 10 examples. The experiment comprised 128 trials, the first 8 of which were warming-up trials and were not included in the analysis. The $8 \times 15$ picture pairs for the four positive and the four negative conditions were presented to the subject in a mixed quasirandom order. For each subject, 15 random sequences of eight picture pairs were constructed, whereby each condition was represented once. Also, which picture pair appeared in any one of the 15 sequences for a given condition was chosen randomly. Each trial was started by the experimenter, and the first click made by the rotating carousal indicated to the subject that the trial had begun. One second later the shutter opened to reveal the picture pair. The pictures remained on the screen until the subject pressed one of the two keys.

\section{Results}

In general, error rates were low, ranging from $0.2 \%$ to $4.2 \%$ in the positive conditions and from $0 \%$ to $1.8 \%$ in the negative conditions, with the exception of the $\mathrm{V}+\mathrm{C}+$ condition, in which the mean error rate was 13.7\%. Error rates paralleled RTs across all conditions, so that a speed-accuracy tradeoff cannot account for the results, which will be described below. Errors were not analyzed further statistically. Extreme RTs that exceeded 2.58 standard deviations from the subject's mean in a given condition were discarded. The means of the remaining RTs in all conditions were then determined for each subject. These scores were then submitted to two separate ANOVAs, one for the positive conditions and one for the negative conditions.

For the positive conditions, the one-way ANOVA (with repeated measures) yielded a significant effect $[\mathrm{F}(3,75)=25.34, \mathrm{MSe}=23,815, \mathrm{p}<.01]$. Post hoc pairwise comparisons using the Newman-Keuls test showed that RTs were significantly shorter under the
Table 1

Mean Reaction Times (in Milliseconds) of Correct Responses in the Negative Trials of Experiment 2 as a Function of Visual Similarity and Conceptual Relatedness

\begin{tabular}{lccc} 
& \multicolumn{3}{c}{$\begin{array}{c}\text { Conceptual Relatedness of the } \\
\text { Depicted Objects }\end{array}$} \\
\cline { 2 - 4 } $\begin{array}{c}\text { Visual Similarity } \\
\text { of the } \\
\text { Pictures }\end{array}$ & $\begin{array}{c}\text { Related } \\
(\mathrm{C}+)\end{array}$ & $\begin{array}{c}\text { Unrelated } \\
(\mathrm{C}-)\end{array}$ & Mean \\
\hline Similar (V+) & 1713 & 1503 & 1608 \\
Dissimilar (V-) & 1479 & 1282 & 1381 \\
Mean & 1596 & 1393 & \\
\hline
\end{tabular}

PIC condition (mean $=1,094 \mathrm{msec}$ ) than under the OBJPERSP condition (mean $=1,242 \mathrm{msec}$ ). The RTs in the latter condition, in turn, were significantly shorter than the RTs under the OBJ-STATES condition (mean = $1,434 \mathrm{msec}$ ) and the BASIC condition (mean $=1,381$ $\mathrm{msec}$ ). The difference between the latter mentioned conditions fell short of significance.

For the negative conditions, a two-way ANOVA (with repeated measures on both factors) indicated that both the visual similarity and the conceptual relatedness between the pictures had a significant effect $[F(1,25)=$ 48.02, MSe $=28,118, \mathrm{p}<.01$, for visual similarity, and $\mathrm{F}(1,25)=49.23, \mathrm{MSe}=21,873, \mathrm{p}<.01$, for conceptual relatedness]. The interaction was not significant $[F(1,25)$ $<1]$. Mean RTs for the four negative conditions are shown in Table 1.

\section{Discussion}

The results confirm the findings of Experiment 1. With a basic-level instruction, the "different" RTs are affected not only by the visual similarity between the stimuli to be compared, but also by the conceptual relatedness. The effect of the latter variation is almost as great as the former: The RTs vary by a mean of $228 \mathrm{msec}$ as a result of the variation of visual similarity, and by a mean of $204 \mathrm{msec}$ as a result of the variation of conceptual relatedness. It can be concluded from our results that, in the comparison of objects, the subjects mentally represented not only the perceptual attributes of the stimuli, but also the nonperceptual attributes that the members of the same superordinate category had in common. Our results contradict those of Murphy and Smith (1982), who found no evidence of the representation of nonperceptual attributes. It is possible, but less likely, that the discrepancy between the two sets of results derives from the difference between the wordpicture matching task used by Murphy and Smith and our picture-picture matching task. Indeed, a word prime is more likely to stimulate the subject's knowledge of the nonperceptual attributes of an object than is a picture prime (Guenther \& Klatzky, 1977). However, it is more plausible that the negative results of Murphy and Smith are linked to the learning phase in their experiment, during which the subjects became accustomed to the stimuli. It may be that, in categorizing stimuli to 
which they had become well-accustomed, the subjects concentrated on the superficial characteristics of the stimuli, so that the "depth of processing" was shallower than with novel stimuli, whose categorization demands a full elaboration of the conceptual representation. The discrepancy between our results and those of Murphy and Smith may also be determined by the differences in material (artificial vs. natural categories), because the functional attributes of objects are related most often to actions and to the pragmatic aspects of a situation. Our knowledge about such attributes normally derives from actual interaction with the object. Perhaps simple verbal information is not enough to instill the knowledge of the functional attributes in such a way that it is spontaneously activated by showing a picture.

Whatever the reasons for the negative findings of Murphy and Smith (1982), our results show that it is incorrect to assume that, in all conditions, subjects mentally represent only perceptual attributes in object identification. Whether or not functional attributes are represented depends, it would seem, on the task.

\section{GENERAL DISCUSSION}

When we began our investigation, our aim was to obtain information about the process of object identification. It seems, however, that picture-picture matching tasks are unsuitable for the study of encoding processes. The RT pattern derived from this task seems to reflect, in whole or in part, the speed of mental comparison and decision processes. If our data are interpreted in this light, then our results suggest, first, that the mental representation of a pictorial stimulus is not always the same. Which attributes of the picture are mentally represented and which are not seems to depend upon the task instruction. Second, it can be inferred from Experiment 2 that, following a basic-level equivalence instruction, not only the perceptual attributes are mentally represented, but also those nonperceptual, functional attributes that members of the same superordinate category have in common.

This summary of our results should be prefaced by some qualifying comments, first with regard to our methodology and the way in which we interpreted the RT. We proceeded from the assumption that, in tasks like these, the RT is an inverse monotonic function of the distance between the subjective similarity of the two stimuli to be compared and the cutoff point. However, our analysis concerning the various coding strategies of the subject groups in Experiment 1 is only valid if we assume this function to be linear. The conclusions we drew would be incorrect if the function were nonlinear, for example, if the change in subjective similarity had an increasingly marked effect on the RT, the nearer it lay to the cutoff point. In that case, we could no longer conclude that the groups represented the stimuli in different ways, because, for example, the RT difference between the OBJ and SUB conditions was greater in the subordinate level instruction group than in the basic- or superordinate-level instruction group; in the subordinate-level instruction group, the variation in similarity was near the cutoff point, whereas in the basic- and superordinate-level instruction groups, the variation occurred at a more distant point on the scale of similarity. In retrospect, the assumption of a linear function appears justified to the extent that in Experiment 2 the effects of the two factors "visual similarity" and "conceptual relatedness" were found to be additive. This suggests that, given a fixed cutoff point, a certain difference in the subjective similarity will always lead to the same, constant difference in the RT, and thus that the RT is actually a linear function of the distance between the subjective similarity of the stimuli and the cutoff point. However, until the assumption of a linear function is proved, our conclusions concerning the task specificity of mental coding must be regarded cautiously.

Some qualifying comments on the way in which we differentiated between attribute types should also be made. Our distinction between "idiosyncratic" and "conceptually relevant" attributes is slightly misleading, in that there are no sharp divisions here. According to studies by Rosch (1978), Tversky (1977), and others, the conceptual relevance of attributes is a matter of degree: An attribute can have more or less cue validity for a category. In this sense, the attributes that we called "idiosyncratic" are to be understood to be those with a minimal cue validity for the conceptual categorization. The comparison of RTs under conditions PIC and OBJ with the RTs under condition SUB shows how far the various instruction groups differentiate between attributes with higher and lower cue validity.

The expression "perceptual attributes" should also be clarified. In Experiment 2, the visual similarity of the stimuli was varied, and the results offer information about the mental representation of those perceptual attributes that are visible or manifest in the stimulus itself. One should distinguish, however, between these manifest attributes and those that, although they are perceptual attributes, are not given in the stimulus. In a black-and-white drawing of a banana, for example, the color yellow is not manifest in the stimulus, but is still a perceptual attribute of the object itself. Our experiments say nothing about such nonmanifest perceptual attributes. An investigation into this question would be both desirable and possible, using picture-picture matching tasks. As a result of the discussion about the basic-level superiority, attention generally has been directed to the differentiation between attribute types (perceptual vs. functional). In most experiments, ours included, attribute type is confounded with perceptibility, because perceptual attributes are for the most part manifest in the stimulus, whereas functional attributes must be inferred. If nonmanifest perceptual attributes were incorporated into the experiment, it would be possible to assess the influence of perceptibility. It might even be the case that the mental repre- 
sentation of an attribute depends more on the perceptibility of an attribute than on its type, perceptual or functional. The significance of the attribute's modality could also be examined: It is probable that, in the process of object identification, nonmanifest visual attributes are more likely to be inferred and mentally represented than, say, are nonmanifest attributes dependent on the senses of hearing or taste. The investigation into the nature of mental representation in object identification would thereby be related to the problems pursued by mental imagery research (cf. Kosslyn, 1981)

\section{REFERENCES}

BABBitT, B. C. (1982). Effect of task demands on dual coding of pictorial stimuli. Journal of Experimental Psychology: Learning, Memory, and Cognition, 8, 73-80.

Barsalou, L. W. (1982). Context-independent and contextdependent information in concepts. Memory \& Cognition, 10 , 82-93.

BARtram, D. J. (1974). The role of visual and semantic codes in object naming. Cognitive Psychology, 6, 325-356.

Bartham, D. J. (1976). Levels of coding in picture-picture comparison tasks. Memory \& Cognition, 4, 593-602.

Durso, F. T. \& Johnson, M. K. (1979). Facilitation in naming and categorizing repeated pictures and words. Journal of Experimental Psychology: Human Learning and Memory, 5, 449-459.

Fraisse, P., Lanati, L., Régnier, J., \& Wahl, M. (1965). Le temps de réaction verbale. II. Réponses spécifiques et catégorielles. Anneé Psychologique, 65, 27-32.

Frost, N. (1972). Encoding and retrieval in visual memory tasks. Journal of Experimental Psychology: General, 95, 317-326.

Guenther, R. K., \& Klatzky, R. L. (1977). Semantic classification of pictures and words. Journal of Experimental Psychology: Human Learning and Memory, 3, 498-514.

Guenthe z, R. K., Klatzky, R. L., \& Putnam, W. (1980). Commonalities and differences in semantic decisions about pictures and words. Journal of Verbal Learning and Verbal Behavior, 19, 54-74.

Herrmann, D. J., Chaffin, R. J. S., Conti, G., Peters, D. \& Robbins, P. H. (1979). Comprehension of antonymy and the generality of categorization models. Journal of Experimental Psychology: Human Learning and Memory, 5, 585-597.

Hoffman, J. (1980, July). Conceptual coding, representation and process. Paper presented at the International Congress of Psychology, Leipzig, GDR.

Hutchinson, J. W., \& Lockhead, G. R. (1977). Similarity as distance: A structural principle for semantic memory. Journal of Experimental Psychology: Human Learning and Memory, $3,660-678$.

Kelter, S. (1982). Conceptual representation of objects in aphasia. Paper presented at the Fifth INS European Conference, Deauville, France.
KLAtzky, R. L., \& Stoy, A. M. (1974). Using visual codes for comparisons of pictures. Memory \& Cognition, 2, 727-736.

KLATZKY, R. L., \& Stoy, A. M. (1978). Semantic information and visual information processing. In J. W. Cotton \& R. L. Klatzky (Eds.), Semantic factors in cognition. Hillsdale, NJ: Erlbaum.

Kosslyn, S. M. (1981). The medium and the message in mental imagery: A theory. Psychological Review, 88, 46-66.

Kroll, N. E. A., Kellicut, M. H., Berrian, R. W., \& KRE ISLER, A. F. (1974). Effects of irrelevant color changes on speed of visual recognition following short retention intervals. Journal of Experimental Psychology, 103, 97-106.

Krueger, L. E. (1978). A theory of perceptual matching. Psychological Review, 85, 278-304.

McCloskey, M., \& Glucksgera, S. (1979). Decision processes in verifying category membership statements: Implications for models of semantic memory. Cognitive Psychology, 11, 1-37.

Mervis, C. B., \& Rosch, E. (1981). Categorization of natural objects. Annual Review of Psychology, 32, 89-115.

MurPhy, G. L., \& SM1Th, E. E. (1982). Basic-level superiority in picture categorization. Journal of Verbal Learning and Verbal Behavior, 21, 1-20.

Posner, M. I., \& Mitchell, R. F. (1967). Chronometric analysis of Classification. Psychological Review, 74, 392-409.

Proctor, R. W. (1981). A unified theory for matching-task phenomena. Psychological Review, 88, 291-326.

RATClifF, R. (1981). A theory of order relations in perceptual matching. Psychological Review, 88, 552-572.

Rosch, E. (1975). Cognitive representations of semantic categories Journal of Experimental Psychology: General, 104, 192-233.

Rosch, E. (1978). Principles of categorization. In E. Rosch \& B. B. Lloyd (Eds.), Principles of categorization. Hillsdale, NJ: Erlbaum.

Rosch, E., Mervis, C. B., Gray, W. D., Johnson, D. M., \& Boyes-Braem, P. (1976). Basic objects in natural categories. Cognitive Psychology, 8, 382-439.

Schaeffer, B., \& Wallace, R. (1970). The comparison of word meanings. Journal of Experimental Psychology, 86, 144-152.

Schvaneveldt, R. W., Durso, F. T., \& Mukherui, B. R. (1982). Semantic distance effects in categorization tasks. Journal of Experimental Psychology: Learning, Memory, and Cognition, 8, 1-15.

Segui, J., \& Fraisse, P. (1968). Le temps de réaction verbale. III. Réponses spécifiques et réponses catégorielles à des stimulus objets. Année Psychologique, 68, 69-82.

SeYmour, P. H. K. (1973). A model for reading, naming and comparison. British Journal of Psychology, 64, 35-49.

Smith, E. E., Shoben, E. J., \& Rips, L. J. (1974). Structure and process in semantic memory: A featural model for semantic decision. Psychological Review, 81, 214-241.

Tversky, A. (1977). Features of similarity. Psychological Review. 84, 327-352.

Warken, C., \& Morton, J. (1982). The effects of priming on picture recognition. British Journal of Psychology, 73, 117-129.

WinER, B. J. (1970). Statistical principles in experimental design. London: McGraw-Hill.

(Manuscript received March 10, 1983; revision accepted for publication October 26, 1983.) 